\title{
Lazy Leukocyte Syndrome—an Enigma Finally Solved?
}

\author{
Amos Etzioni $^{1}$ (D) Hans D. Ochs ${ }^{2}$ \\ Received: 25 June 2019 / Accepted: 4 November 2019 / Published online: 25 November 2019 \\ (C) Springer Science+Business Media, LLC, part of Springer Nature 2019
}

\section{The Disease of Lazy Leukocytes}

The initial report in the Lancet [1] described two unrelated children, a 5-year-old girl and a 2-year-old boy, with a history of early onset gingivitis, stomatitis, and recurrent otitis media. The family histories were unremarkable. Both children had severe neutropenia with normal bone marrow examination. Immunological parameters, including serum immunoglobulin levels and lymphocyte responses to phytohemagglutinin, were normal. While phagocytosis and bactericidal activity were similar to controls, the migration of the patients' neutrophils was markedly defective, both in vivo (by Rebuck skin window) and in vitro (using a modified Boyden chamber). The fact that both random migration and chemotaxis were defective pointed to an intrinsic leukocyte defect and raised, in the authors' opinion, the possibility of a membrane defect in patient neutrophils [1].

Several years later, in 1975, another child with early onset stomatitis, purulent skin lesions and recurrent episodes of bronchopneumonia was reported to have abnormal neutrophil chemotaxis associated with persistent neutropenia [2]. A third case of LLS, published in 1978, was observed in a 40-year-old woman who suffered since early childhood from gingivitis, skin infections and upper respiratory tract infections associated with persistent neutropenia [3]. Scanning electron microscopy of the patient's neutrophils showed alterations in the cell surface configuration, characterized by coarsening of the normally fine ruffles and the appearance of knob-like projections. Based on these findings, the authors suggested that LLS may be due to dysfunction of the microtubular proteins in the neutrophil membrane, leading to excessive rigidity and thus

Amos Etzioni

Etzioni@rmc.gov.il

1 Ruth Children Hospital, Rambam Medical Campus and the Faculty of Medicine, Technion, Haifa, Israel

2 University of Washington and Seattle Children's Research Institute, Seattle, WA, USA defective neutrophil migration. In the same year, Gallin et al. described a 7-year-old girl with recurrent life-threatening bacterial infections starting at the age of 3 months who had markedly abnormal random and directed chemotaxis with normal neutrophil counts, and who died at the age of 8 years of disseminated varicella [4]. In 1984, another possible case of LLS was reported in a 35-year-old woman with neutropenia and abnormal distribution of actin in neutrophils [5].

Analysis of archived DNA from Gallin's patient, performed some 40 years after her death, revealed a compound heterozygous mutation in WDR1 [6], the gene encoding the Actin-interacting-protein 1 (AIP1) which plays an important role in actin depolymerization [7].

\section{The Pathogenesis}

After these few reports no additional patients with LLS were published in the literature and the syndrome of lazy leukocytes became a forgotten malady. This may in part be explained by the lack of standardized techniques to assess direct and random mobility. A breakthrough came in 2016 when Kuhns et al. [6] provided evidence that patients resembling those described by Miller and colleagues may have had a defect in actin cytoskeleton regulation similar to those with mutations in WDR1 or other genes associated with actinopathies.

Dynamic reorganization of the actin cytoskeleton is necessary for several biological processes including rapid and directional actin remodeling required for leukocyte migration [7]. The importance of the actin cytoskeleton is highlighted by immune deficiency conditions due to inborn errors in one of its multiple components [8,9]. Morphologic changes in leukocytes are required for spreading, and for migration through the endothelium into the tissues. These changes will alter the fluidity of the cytosol from gel to sol and back, a process depending on the dynamic balance between the two forms of actin, the monomeric form, called globular $(\mathrm{G})$ actin, and its filamentous form, F-actin. The latter is responsible for generating the branched network that supports the formation 
of lamellopodia, which are essential for leukocyte migration [10]. Since polymerized F-actin is relative stable, several specific actin regulator proteins are required to promote actin filament disassembly [11].The actin depolarizing factor (ADF), also called cofilin, promotes actin disassembly by severing actin filaments and dissociating actin monomers from filaments [11]. It was subsequently shown that additional proteins play an important role in this process. AIP1 contributes to actin filament dissociation by binding to cofilin and thus enhancing its actin depolarizing activity [12]. AIP1 induces a conformational change in cofilin that results in enhanced filament severing activity. The importance of AIP1 for neutrophil locomotion was first observed in mice carrying 2 hypomorphic alleles of $\mathrm{Wdr} 1$ resulting in a macrophage maturation defect and macrothrombocytopenia [13]. Neutrophils from mutant mice exhibited defective chemokine induced actin reorganization and abnormal chemotaxis. In addition, WDR1-deficient mice developed an autoinflammatory disease characterized by a massive infiltration of neutrophils into inflammatory lesions [13] which was associated with enhanced release of IL-18 but not IL-1beta [14]. In mice loss of function mutations in Aip1 are embryotic lethal [13].

In 2016 Kuhns et al. [6] described 4 children from 3 unrelated families with biallelic mutations in WDR1 whose clinical phenotype resembled in part that described by Miller et al. [1]. The three girls and one boy presented with severe, in one case fatal, disease characterized by persistent stomatitis leading to oral stenosis requiring placement of a gastrostomy tube, recurrent respiratory tract infections, and mild neutropenia. Chemotaxis was markedly impaired in all patients, while bacterial killing and oxidative burst were intact. Neutrophil Factin was elevated fourfold. In one patient allogeneic stem cell transplantation (HSCT) corrected the neutrophil defect [6]. No heterozygous phenotype was observed and all missense mutations occurred at highly conserved regions considered to be important for AIP1 function.

The authors concluded that, as overall growth and development were normal in these patients, AIP1's major role is to rapidly remodel the cytoskeleton, a process intrinsic mainly to leukocyte function [6]. This is in agreement with the observation that AIP1 is expressed in most types of leukocytes, but not in skeleton muscles.

\section{The Surprise}

Based on the initial report [6] the neutrophil defect was considered to be the predominant clinical condition associated with mutations in WDR1. However, in 2017, Standing et al. [15] described two sisters, born to consanguineous parents, with a mutation in WDR1, and a strikingly different clinical phenotype. Starting early in life they suffered from autoinflammatory periodic fever, occurring every 2 months and lasting up to a week. Both had a history of recurrent oral inflammation, recurrent pneumonias and other bacterial infections and chronic thrombocytopenia, but defects in adaptive immunity could not be demonstrated. The girls responded only partially to steroids and their condition deteriorated. One died at 14 years of age from "sterile systemic inflammation" while the other underwent successful HSCT. Using whole exome sequencing, a homozygous missense mutation was identified in the WDR 1 gene, in line with the increased level of polymerized F-actin in patient-cells. Both patients had high serum level of IL-18 which seems to play an important role in the pathogenesis of the autoinflammatory condition observed in Wdr1-deficient mice [14].

Recently, Pfajfer et al. reported yet another set of symptoms as a consequence of mutations in the WDRl gene [16]. By detailed assessment of the adaptive immune system in their cohort of 6 patients from 3 unrelated consanguineous families with biallelic WDR1 mutations, a broadly defective B cell compartment was demonstrated including profound $\mathrm{B}$ cell lymphopenia associated with low B cell precursors in the bone marrow. Circulating B cells were predominantly naïve and not isotype switched, and exhibited reduced clonal diversity. Serum immunoglobulin levels and specific antibody titers were within the normal range in most patients, although the majority were on IVIG replacement therapy. T cell deficiency, on the other hand, was mild and limited to reduced numbers of follicular T-helper cells and low TCR-mediated activation to anti-CD3. As had been reported previously $[6,15]$, neutrophil abnormalities were consistent and included nuclear herniation, accumulation of cytoplasmatic F-actin, and impaired migration in response to fMLF [16]. However, in contrast to the original report of AIP1 deficiency [6] and the historic descriptions of LLS patients [1-5], neutrophil counts in subsequently reported patients were persistently normal or elevated $[15$, $16]$.

In conclusion, the entity aptly named lazy leukocyte syndrome based on abnormal in vivo and in vitro chemotaxis of phagocytes can now be added to a group of disorders caused by single gene defects resulting in impaired cytoskeletal dynamics, referred to as actinopathies (Table 1). Based on syndromic features other than infections, LLS can be clearly differentiated from other actinopathies such as the WiskottAldrich syndrome (WAS), X-linked neutropenia (XLN) caused by gain of function (GOF) mutation in the cdc42 binding site of the WAS gene, WAS interacting protein (WIP) deficiency, DOCK 8 deficiency, RASGRP1 deficiency, CORONIN-1A deficiency, DOCK2 deficiency, Rac2 deficiency, beta-Actin deficiency, and ARPC1B deficiency (Table 1) (17-20). Actinopathies differ from leukocyte adhesion defects (LAD1, 2, 3) based on the characteristic LAD findings of neutrophilia and lack of pus formation. While it is unlikely that all cases resembling the definition of LLS have a mutation in WDRl, it is intriguing to speculate that Spike 


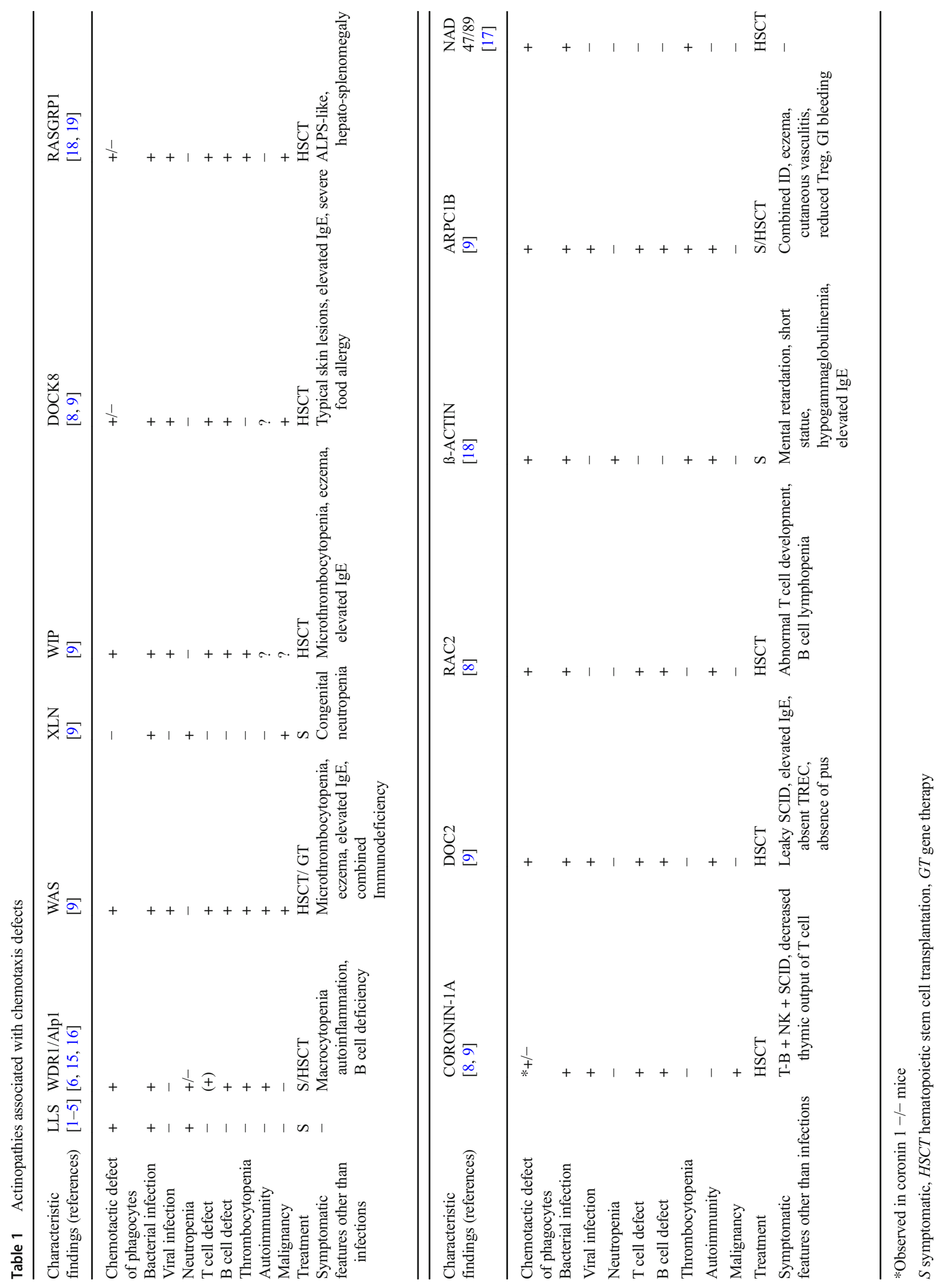


Miller's 1971 LLS paper could be the first description of the recently designated actinopathies, demonstrating the power of sophisticated immunologic and genetic testing that deciphered the molecular underpinning of a syndrome with highly variable symptoms, caused by mutations in WDR1/AIP1. This hypothesis would explain the evolution of a clinical observation, made by an astute clinician half a century ago, to a continuously expanding phenotype characterized by defective leukocyte mobility, abnormal adaptive immunity, and symptoms of autoinflammation.

\section{Compliance with ethical standards}

Conflict of Interest The authors declare that they have no conflict of interest.

\section{References}

1. Miller ME, Oski FA, Harris MB. Lazy leukocyte syndrome a new disorder of neutrophil function. Lancet. 1971;1(7701):665-9.

2. Constantopoulos A, Karpathios T, Nicolaidou P, Maounis F, Matsaniotis N. Lazy leukocyte syndrome. J Pediatrics. 1975;87(6):945-5.

3. Pinkerton PH. RobinsonJB, Senn JS. Lazy leukocyte syndromedisorder of the granulocyte membrane? J Clin Pathol. 1978;31(4): $300-8$.

4. Gallin JI, Malech HL, Wright DG, Whisnant JK, Kirkpatrick CH. Recurrent severe infections in a child with abnormal leukocyte function: possible relationship to increase microtubule assembly. Blood. 1978;51(5):919-33.

5. Goldman JM, Foroozanfar N, Gazzard BG, Hobbs JR. Lazy leukocyte syndrome. J Royal Soc Med. 1984;77(2):140-1.

6. Kuhns DB, Fink DL, Choi U, Sweeney C, Lau K, Long Priel D, et al. Cytoskeletal abnormalities and neutrophil dysfunction in WDR1 deficiency. Blood. 2016;128(17):2135-43.

7. Ono S. Functions of actin-interacting protein 1 (AIP1)/ WD repeat protein 1 (WDR1) in actin filament dynamics and cytoskeletal regulation. Biochem Biophys Res Commun. 2018;506(2):315-22.

8. Tangye SG, Bucciol G, Casas-Martin J, Pillay B, Ma SS, Moens L, et al. Human inborn error of the actin cytoskeleton affecting immunity: way beyond WAS and WIP. Immunol Cell Biol. 2019;97(4): 389-402.
9. Janssen E, Geha RS. Primary immunodeficiencies caused by mutations in actin regulatory proteins. Immunol Rev. 2019;287(1): $121-34$.

10. Southwick FS. The lazy leukocyte syndrome revisited. Blood. 2016;128(17):2112-3.

11. Bamburg JR. Proteins of the ADF/cofilin family: essential regulators of actin dynamics. Ann Rev Cell Dev Biol. 1999;15:185-230.

12. Ono S. Regulation of actin filament dynamics by actin depolymerizing factor/cofilin and actin interacting protein 1: new blades for twisting filaments. Biochemistry. 2003;42(46):1336370.

13. Kile BT, Panopoulos AD, Stirzaker RA, Hacking DF, Tahtamoum LH, Willson TA, et al. Mutations in the cofilin partner Aip1/WDR1 cause autoinflammatory disease and macrothrombocytopenia. Blood. 2007;110(7):2371-80.

14. Kim ML, Chae JJ, Park YH, De Nardo D, Stirzaker RA, Ko HJ, et al. Aberrant actin depolarization triggers the pyrin inflammasome and autoinflammatory disease that is dependent on IL-18, not IL1beta. J Exp Med. 2015;212(6):927-38.

15. Standing ASI, Malinova D, Hong Y, Record J, Moulding D, Blundell MP, et al. Autoinflammatory periodic fever, immunodeficiency, and thrombocytopenia (PFIT) caused by mutation in actinregulatory gene WDR1. J Exp Med. 2017;214(1):59-71.

16. Pfajfer L, Mair NK, Jimenez-Heredia R, Genel F, Gulez N, Ardeniz $\mathrm{O}$, et al. Mutatoins affecting the actin regulatory WD repeat containing proetein 1 lead to aberrant lymphoid immunity. J Allergy Clin Imunol. 2018;142(12):1589-604.

17. Coates TD, Torkidson JC, Torres M, Church JA, Howard TH. An Inherited defect of neutrophil motility and microfilamanetous cytoskeleton associated with abnormalities in $47-\mathrm{Kd}$ and $89-\mathrm{Kd}$ proteins. Blood. 1991;78(5):1338-46.

18. Mao H, Yang W, Latour S, Yang J, Winter S, Zheng J, et al. RASGRP1 mutation in autoimmune lymphoproliferative syndrome -like disease. J Allergy Clin Immunol. 2018;142(2):595-604.

19. Salzer E, Cagdas D, Hons M, Mace EM, Garncarz W, Petronczki OY, et al. RASGRP1 deficiency causes immunodeficiency with impaired cytoskeletal dynamics. Nat Immunol. 2016;17(12): 1352-60.

20. Nunoi H, Yamazaki T, Tsuchiya H, Kato S, Malech HL, Matsuda I, et al. A heterozygous mutation of beta-actin associated with neutrophil dysfunction and recurrent infection. Proc Nat Acad Sci. 1999;96(15):8693-8.

Publisher's Note Springer Nature remains neutral with regard to jurisdictional claims in published maps and institutional affiliations. 